\title{
An adaptation level theory of tinnitus audibility
}

\author{
Grant D. Searchfield ${ }^{1,2 *}$, Kei Kobayashi ${ }^{1}$ and Michael Sanders ${ }^{1}$ \\ Audiology Section and Centre for Brain Research, The University of Auckland, Auckland, New Zealand \\ ${ }^{2}$ Tinnitus Research Initiative, Regensburg, Germany
}

\author{
Edited by: \\ Larry Roberts, McMaster University, \\ Canada \\ Reviewed by: \\ Dirk De Ridder, University Hospital \\ Antwerp, Belgium \\ Pawel J. Jastreboff, Emory University \\ School of Medicine, USA \\ *Correspondence: \\ Grant D. Searchfield, Audiology \\ Section, The University of Auckland, \\ Private Bag 92109, Auckland, New \\ Zealand. \\ e-mail: g.searchfield@auckland.ac.nz
}

\begin{abstract}
Models of tinnitus suggest roles for auditory, attention, and emotional networks in tinnitus perception. A model of tinnitus audibility based on Helson's (1964) adaptation level theory (ALT) is hypothesized to explain the relationship between tinnitus audibility, personality, memory, and attention. This theory attempts to describe how tinnitus audibility or detectability might change with experience and context. The basis of ALT and potential role of auditory scene analysis in tinnitus perception are discussed. The proposed psychoacoustic model lends itself to incorporation into existing neurophysiological models of tinnitus perception. It is hoped that the ALT hypothesis will allow for greater empirical investigation of factors influencing tinnitus perception, such as attention and tinnitus sound therapies.
\end{abstract}

Keywords: tinnitus, adaptation level, treatment, model

\section{INTRODUCTION}

Tinnitus is thought to be the result of a cascade of events in the auditory pathways, often commencing with injury to the auditory periphery (Baracca et al., 2011; Zhou et al., 2011). After a peripheral lesion, the pattern of sensory input to the auditory cortex changes (Martin, 1995; Searchfield et al., 2004) believed to result in the brain adapting to new patterns of activity at subcortical (Kaltenbach et al., 2005) and cortical centers (Eggermont and Roberts, 2004) resulting in the perception of sound. Brain networks with elements not classically considered part of the auditory system then contribute to, or drive, the awareness and severity of tinnitus (Zenner et al., 2006; De Ridder et al., 2011). The severity of tinnitus is likely to be determined by a multi-layered process that involves auditory, attention, and emotional networks (Jastreboff, 1990; Kaltenbach, 2006; Zenner et al., 2006; De Ridder et al., 2011).

The majority of studies investigating tinnitus processing have approached it from a neuroanatomical or physiology tradition (Roberts et al., 2010) often using pain as an analogy (Moller, 2000). An additional approach is to consider tinnitus from a psychoacoustical perspective (Penner and Bilger, 1995) in which audibility is governed by context, memory, attention, and personality (Welch and Dawes, 2008). The process that the auditory system uses to identify and differentiate auditory objects has become known as auditory scene analysis (ASA; Bregman, 1990). In this paper we introduce an empirically testable model of tinnitus audibility based on Helson's (1964) adaptation level theory (ALT) and we discuss potential roles for ASA in differentiating tinnitus from ongoing auditory activity, consistent with previous models describing pattern recognition roles in tinnitus perception (Jastreboff, 1990). Tinnitus audibility is defined here as a stimulus dimension to which many variables contribute to tinnitus being heard. Tinnitus loudness is one, but not the only, contributing factor to its audibility.

The multi-dimensional nature of tinnitus is well demonstrated by the incongruence between self-report of tinnitus magnitude and psychoacoustic intensity matches (Baskill and Coles, 1999).
A person who suffers from tinnitus may report it as sounding very loud, but match it to a low-intensity external sound (Jakes et al., 1986). It is also known that the common report of tinnitus is far more frequent than complaints of tinnitus effects on quality of life (Gopinath et al., 2010). Such observations are consistent with models of tinnitus distress that identify it not solely as an auditory phenomenon, but a process incorporating emotion and reaction (Jastreboff, 1990; Zenner et al., 2006; De Ridder et al., 2011). The pioneering work of Heller and Bergman (1953) suggested that spontaneous auditory perceptions occur in a majority of people given an ideal signal detection context. Perception of tinnitus-like sounds occurs in between 64\% (Tucker et al., 2005) and 94\% (Heller and Bergman, 1953) of people without tinnitus when listening in a silent environment. This "tinnitus" audibility is governed to some degree by attention (Knobel and Sanchez, 2008). Prolonged auditory deprivation (through ear-plug use) is associated with an increase in sensitivity to sound possibly through an adaptive plasticity process leading to centrally mediated increase in gain; opposite effects are seen with sound stimulation (Formby et al., 2003). Such a mechanism may also be responsible for reduced tinnitus complaint with long-term stimulation with low-level sound (Norena, 2011). Tinnitus loudness, alone, does not determine severity but a multi-dimensional concept of tinnitus audibility, expressed by ALT, may have ramifications for treatments that incorporate sound to reduce audibility or detection of tinnitus.

\section{ADAPTATION LEVEL THEORY}

The complex processing responsible for tinnitus perception (Zenner et al., 2006; De Ridder et al., 2011) can be sub-served by a simple psychoacoustical model of audibility that accounts for auditory context, attention, and individual factors such as memory and personality. Helson's (1964) ALT has been widely used in sensory perception and psychophysics to explain magnitude adjustments to context (Murch, 1973; Coren and Ward, 1989; Gescheider, 1997). Adaptation level effects and similar "differential contextual effects" (Marks and Arieh, 2006) have been demonstrated 
in perception of: touch, taste, olfaction (Marks and Arieh, 2006), pain (Rollman, 1979; Kyle et al., 2009), weight (Helson, 1948), temperature (Masuyama, 1994), loudness (Marks, 1994), vision (Helson, 1964), phobias (Lauterbach, 1979), and even market research (Della Bitta and Monroe, 1974). There are a number of overlapping terms to describe change in perception with repeated exposure including acclimatization, adaptation, stimulus failure, fatigue as well as habituation (Helson, 1964; Mazess, 1975; McBurney and Balaban, 2009). The use of the terms are often governed by the training of the author, for example, adaptation is often used in physiology to describe a change, possibly short-term, in the response of sensory systems following stimulation, habituation is a decrease in response after repeated stimulation, sensitization an increase in response with repeated stimulation. In many other fields adaptation is a general term indicating broad effects across all biological and social levels (Helson, 1964; Mazess, 1975), for example, affective adaptation involves psychological processes that reduce responses to emotional events (Wilson and Gilbert, 2008). Adaptation is a two-way process allowing both an increase or decrease in response (Helson, 1964). Consistent with its original use by Helson "adaptation" is used here to refer to change, but does not ascribe a physiological mechanism of change.

Adaptation level theory does not appear to have been applied to tinnitus before, but potentially explains some of the unusual psychoacoustic characteristics of tinnitus as well as appearing as a means to quantify the contrast between signal and background noise thought to be important for tinnitus "sound therapy."

Sound therapy is aimed at facilitating the process of habituation of both tinnitus-induced reaction and tinnitus perception by decreasing the difference between tinnitus-related neuronal activity and background neuronal activity. Since all our senses work on the basis of differences between signals and background, and not on its absolute value, by decreasing the difference between the tinnitus signal and the background neuronal activity it is easier for the central nervous system to filter out or block tinnitus-related activity. (Jastreboff, 1999b, p. 491)

Adaptation level theory is a longstanding psychoacoustic theory commonly used to explain and quantify the differences in signal and background described by Jastreboff above. The basis of ALT is that no stimulus can be understood in isolation (Helson, 1964). The adaptation level is an anchor or reference point for sensory magnitude and discrimination (Helson, 1964; Coren and Ward, 1989). The strength of a stimulus is compared to the central point of reference (the adaptation level), sensations below the ALT are less, above greater, the larger the distance between the adaptation level and stimulus the stronger it is (Broadbent and Ladefoged, 1960; Lauterbach, 1979). Judgments of tinnitus magnitude are hypothesized to change over time according to situation, attention, and psychological factors. The adaptation level is the combined effects of present and past experience and is the level to which comparisons are made (Helson, 1964; Della Bitta and Monroe, 1974).

Helson (1964) postulated that the adaptation level was a weighted product of three components (external and internal): (1) Focal stimuli, (2) background or contextual stimuli, and (3) residuals. The focal stimuli are those being attended to, the background stimuli are the context, and the residual stimuli are the sum of factors such as past experience (i.e., memory) physiological state (arousal), and personality (Helson, 1964; Murch, 1973). We suggest that contributions of residuals, focal, and background stimuli need to be considered in judgment of tinnitus, along with the role of attention in emphasizing each elements importance. Helson $(1964$, p. 58) proposed that his ALT could be mathematically expressed simply as:

\section{$A=X^{\mathrm{p}} B^{\mathrm{q}} R^{\mathrm{r}}$}

Where $A$ is the adaptation level, $X$ is the geometric mean of the focal stimuli, $B$ is the background stimuli, and $R$ are residuals (memory, arousal, and personality). The weighting coefficients $p$, $q$, and $r$ determine the relative contributions to adaptation level. In its application to tinnitus we propose $A$ represents the adaptation level of tinnitus (audibility) in the environment, $X$ is the focal stimulus (tinnitus) magnitude, $B$ is background sound (e.g., sound therapy) magnitude, $R$ are residuals such as personality, and the weighting factors are related to attention and ASA. In the following discussion we suggest how these three components interact to create the tinnitus percept.

\section{FOCAL ( $X$ ) AND BACKGROUND (B) STIMULI}

The processes involved in tinnitus perception are likely to be similar to the analysis of complex sounds (Zenner et al., 2006). ASA is the process in which we try to make sense of our soundscape and solve the "the cocktail party" problem of extracting important sounds from background noise (Cherry, 1953; Winkler et al., 2009; McLachlan and Wilson, 2010). Tinnitus may be considered the focal or stimulus of interest, while background noises are competitive stimuli. ASA consists of at least two processes: a primitive process based on signal segregation, and a schema-based learning process (Bregman, 1990; Alain and Arnott, 2000). The process of sound object perception requires memory, attention as well as processing of the auditory signal; many of these processes have recently come into focus for the generation and maintenance of tinnitus (Zenner et al., 2006; Haab et al., 2009; De Ridder et al., 2011).

Griffiths and Warren (2004) proposed four general principles of object analysis that can also be applied to analysis of tinnitus:

1. Analysis of information from the sensory world.

2. Separation of auditory object (tinnitus) from sensory world.

3. Extraction and generalization of sensory information within the same dimension (audition).

4. Generalization between senses.

Tinnitus is unusual in that its internal representation conflicts with our sensory expectations and ability to generalize to experience. Tinnitus is perceived as a distinct auditory object (Principle 1) patients report specific tinnitus sounds and seek to identify their source (Feldmann, 1992). Tinnitus is recognized as comprising a combination of frequency, intensity, and temporal patterns that enable the individual to differentiate between tinnitus' auditory signature against other sounds (Principle 2) so successful is the 
auditory system at this task probably aided by attention (see discussion on weighting factors) and Gestalt-like grouping [similar to figure-ground in visual perception (Qiu and Von Der Heydt, 2005)] that masking may not be achievable (Feldmann, 1971). Tinnitus appears to "pop out" from environmental sound; this effect is also seen for sounds that are incongruent with the soundscape (Leech et al., 2009; Fishman and Steinschneider, 2010). Past experience may shape object formation. Audibility of tinnitus probably requires encoding and analysis of repeating patterns or "predictive regularity" (Winkler et al., 2009). Mismatch between activity and expectations (memory) of auditory object representation (such as multi-sensory interaction to confirm source of sounds, e.g., vision and touch) may underpin Feldman's (1992) description of tinnitus as having a different reality to normal auditory objects.

The mechanisms underpinning interaction of sound with tinnitus (Principle 3) are not well understood. True sounds can cover (mask) each other by reducing probability of detection, interfere with different identifying features (example pitch), or disruption of meaning (Kidd et al., 2002). There are two general mechanisms of auditory masking. One mechanism, "energetic" masking, occurs when one sound interferes with the process of encoding another sound at the level of the inner ear and auditory nerve, such as when the basilar membrane traveling wave of the masker obscures or "swamps" that of the signal (Kidd et al., 2002; Scott et al., 2004). The other mechanism of sound masking occurs when a sound of interest cannot be extracted from another on the basis of its content or cognitive load; this is a central process of masking and has been termed "informational" masking (Oh and Lutfi, 1999; Kidd et al., 2002; Durlach et al., 2003; Scott et al., 2004; Watson, 2005; Gutschalk et al., 2008; Leech et al., 2009). Tinnitus masking is believed to be the result of central processing (Penner, 1987) as: (1) it does not always occur in a frequency specific manner, (2) masking sound presented to the ear opposite the perceived location of tinnitus can successfully mask tinnitus, and (3) tones may mask tinnitus described as a broadband sound (Feldmann, 1971). However, the precise mechanism of tinnitus masking remains uncertain. The central auditory masking effect known as informational masking may, along with other mechanisms such as suppression (Jastreboff, 1999b), contribute to reducing tinnitus detectability in sound. In informational masking the signal may be represented, but detection is affected because of competition for limited processing capacity between signal and masker (Watson, 2005). For example, competing speech makes hearing conversation difficult because of the information it contains, as well as any masking from overlapping spectral characteristics. Informational masking is likely to be influenced by attention and could be considered a weighting factor for tinnitus audibility in our ALT model of tinnitus.

The generalization of tinnitus (Principle 4) may lead to a conflict between tinnitus and our expectations for an auditory object; this conflict is possibly crucial to explain the annoyance and attention paid to tinnitus (Feldmann, 1992; De Ridder et al., 2011). Unlike true sounds tinnitus does not have a source that can be seen, touched or correlated to other sensory input (Feldmann, 1992). This conflict with the environment and memory of true sound characteristics may trigger adaptive tuning (Grossberg et al., 2004), attention, and inhibitory mechanisms that would normally sub-serve a role in improving sound source identification, consequently further driving attention to the tinnitus perception.

The level of sound in the environment affects tinnitus perception (Heller and Bergman, 1953) this observation is important for understanding the role of ALT in the psychoacoustics of tinnitus. Most psychoacoustic evaluations are undertaken in the very quiet environment of sound treated audiological booths, quite unlike the normal soundscape of the individual with tinnitus. Tinnitus sufferers will report, and magnitude estimations support, tinnitus as a loud intrusive sound, however, psychoacoustical tinnitus loudness matches are low, rarely greater than $20 \mathrm{~dB}$ above threshold (Penner, 1986). Attempts to explain the difference between the sufferers rating of tinnitus magnitude and loudness match to external sounds have centered on loudness recruitment (Penner, 1986). Loudness recruitment occurs in the presence of hearing loss and is an elevation in threshold without an increase in loudness discomfort levels; this results in a reduced dynamic range and more rapid growth of loudness (Penner, 1986). Studies attempting to accommodate for recruitment in tinnitus loudness match have provided mixed results (Penner, 1986). Henry and Meikle (1996) undertook monaural and binaural measures of loudness growth at both reference and tinnitus frequencies. Variability in loudness match that was attributed to loudness growth was only $25 \%$. Consequently much of the paradoxical loudness of tinnitus remains unexplained (Henry and Meikle, 1996). ALT potentially explains some of the paradox, as it has in pain perception research. Pain is often used as a model for tinnitus (Moller, 2000). Patients with chronic pain have higher thresholds for unpleasantness, and experimental pain stimuli (e.g., electrical stimulation) are less intense and unpleasant than in pain free volunteers (Rollman, 1979; Boureau et al., 1991). According to ALT persons experiencing chronic pain have higher internal anchor points for pain, which lowers the subjective severity of induced experimental pain (Rollman, 1979; Boureau et al., 1991).

If these findings are applied to tinnitus some of the variability between psychoacoustic loudness matches and magnitude estimations may be explained. The experimental condition and simple addition of a comparison sound can bias the adaptation level. In magnitude estimations tinnitus is usually compared to the quiet environment of the consultation room or research laboratory. Loudness matches using an external matching sound are not compared to the absence of sound, but instead to a new adaptation level which incorporates the test stimulus with the existing reference point of tinnitus. The relationship between the stimulus (matching sound) and prevailing level (tinnitus) determines its perceived magnitude and quality. The mere presence of a comparison stimulus alters the adaptation level, creating a new anchor point so that tinnitus is matched to an external matching sound at a level that is lower than anticipated.

\section{RESIDUALS ( $R$ )}

The residual component of Helson's theory consists of psychological and individual factors that can influence perception. Memories of sound, past experience, arousal level, and personality will have a strong or negligible effect on tinnitus depending on the individual residuals influencing factors such as an individual's 
signal detection criterion (Welch and Dawes, 2008). Memories and past experience may prime tinnitus and sound therapy effects. The detection and maintenance of tinnitus in the sensory and cognitive domains will interact with individual differences in health, coping, acceptance, motivation, and personality to determine magnitude of response (Helson, 1964; Revelle, 1995). If tinnitus is seen as being behaviorally important (Jastreboff and Hazell, 2004) or perceived out of context, conflicting with reality (Feldmann, 1992) it will take on greater importance relative to other sounds. A loss of control over the environment, such as the uncontrollable experience of sounds that have no auditory source in the environment (tinnitus), may lead to learned helplessness including a reduction in coping behaviors (Overmier, 2002). Welch and Dawes (2008) found that, in a population sample of 32 year olds, those who experienced tinnitus were less close to others, less constrained, and more negatively emotional. It was concluded that personality traits biased tinnitus reporting and influenced tinnitus signal detection (Welch and Dawes, 2008).

\section{WEIGHTING FACTORS $(p, q$, AND $r)$}

Increasing evidence points toward the role of attention in tinnitus perception (Cuny et al., 2004). In ALT, attention has an important role in determining focus and possibly weighting of different components to the adaptation level. Alertness and orientation effects are likely to affect the perception of loudness (Stallen, 2008). The sound levels in different environments (busy office, quiet bedroom, and party) can increase or decrease audibility of tinnitus in a manner predicted by ALT. If there is a reduction in background sound levels or a change in focus there will be a greater weighting to tinnitus. In an interesting addition to the classic Heller and Bergman (1953) experiment of listening for sound in quiet, Knobel and Sanchez (2008) manipulated attention while keeping background sound constant. When simply listening for sounds $68.2 \%$ of individuals heard tinnitus, when involved in a visual attention task this dropped to $45.5 \%$, and only $19.7 \%$ experienced hearing sounds in silence when completing a stacking task ("Tower of Hanoi"; Knobel and Sanchez, 2008). Interpreting the results in an ALT framework the focus ( $X$, tinnitus) and background ( $B$, silence) were constant, as were residuals $(R)$ for the given individual, but the weighting factors ( $p, q$, and $r$; attention and arousal) varied. Greater cognitive load reduced tinnitus perception. In situations where attention and higher executive function are directed to non-tinnitus activities (e.g., work) processing of tinnitus may take a lower priority to that required when in a low-demand situation (e.g., relaxing at home at the end of a hard days work). Cognitive resources are needed to maintain distinction between target and distractor (Lavie, 2005); attention load on non-auditory activities is less likely to see emergence of tinnitus perceptions (Knobel and Sanchez, 2008). Detection and attention are strongly intertwined and the unusual percept of tinnitus may become a magnet to attention and draw resources from useful cognitive processing to detrimental processing of tinnitus; explaining reduced cognitive performance amongst sufferers (Andersson et al., 2000; Cuny et al., 2004). It is a common clinical observation that tinnitus patients will say the tinnitus is not a problem when they are busy. Involvement in non-tinnitus focused activities such as work, hobbies and exercise, may change how and if people react to the tinnitus percept. Tinnitus counseling, psychological-based treatments, and simple attention training (Henry and Wilson, 2002) may reduce the weighting on residual factors, contributing positively to reductions in tinnitus magnitude.

\section{MECHANISMS OF "ADAPTATION"}

Like ASA and streaming (Griffiths and Warren, 2004; Snyder and Alain, 2007), tinnitus is probably the result of complex interactions at multiple levels of the auditory system. Tinnitus and sound organization may begin as early as the cochlear nucleus (Kaltenbach, 2006; Pressnitzer et al., 2008; Brozoski et al., 2012) with the auditory cortex (Micheyl et al., 2007; Roberts et al., 2010) and information processing centers (Giraud et al., 1999; Griffiths and Warren, 2002, 2004) playing important roles. Detection of auditory targets (such as tinnitus) can be considered to consist of three central processes and associated brain topography: perceptual discrimination, interaction between frontal and temporal regions; stimulus-response association, temporo-parietal regions; post-perceptual processing, right temporo-parietal region (Shahin et al., 2006). Following detection tinnitus may become a problem due to multiple overlapping networks one of which is a distress network (consisting of the anterior cingulate cortex, amygdale, and anterior insular) with memory mechanisms playing a role in awareness of tinnitus and reinforcement of distress (De Ridder et al., 2011).

Adaptation level theory is a psychophysical theory and does not attempt to ascribe a physiological mechanism to change in tinnitus, but it can accommodate many existing models. There are many potential mechanisms which could account for a changed audibility of tinnitus over different timescales including habituation (Jastreboff, 1999a), reversal of cortical reorganization (Okamoto et al., 2010), and gain related processes (Norena, 2011). The perception of tinnitus in therapeutic sound is, perhaps, analogous to hearing sounds of interest in background noise. The ability to hear target sounds in noise, such as tinnitus, may involve habituation to the noise and also, possibly, bottom-up driven attention, refractoriness, and stimulus-specific adaptation (Lagemann et al., 2010) and top-down process such as selective attention (Jacobson et al., 1996; Alain and Arnott, 2000). Stimulus-specific adaptation effects appear to occur throughout the auditory pathways (Robinson and McAlpine, 2009) from early auditory processing (Marks and Arieh, 2006) to the auditory cortex (Micheyl et al., 2007; Rabinowitz et al., 2011). Neurons in the auditory system require some form of adaptation system to cope with the dynamic range of sounds and changes in the environment over time (Dean et al., 2005, 2008). Dean et al. (2005) demonstrated that individual neurons in the inferior colliculus of guinea pigs adjusted to stimulus statistics (such as the mean) of the most commonly occurring sounds, possibly through spike frequency adaptation (a decline in the frequency of firing over time with constant stimulation). Robinson and McAlpine (2009) believe that shifts in response range are determined by stimulus statistics and contextual importance (such as prior experience); which is consistent with Helson's (1964) ALT. Change in tinnitus could be due to such alterations in the underlying distribution of neural activity or decrease in criteria set for detection (Welch and Dawes, 2008). According to Welch and Dawes (2008) theory the placement of signal detection 
criterion reflects the individual's predisposition to detect a given level of activity. Two people with the same injury related neural activity may or may not experience tinnitus on the basis of this signal detection criterion. The criterion may differ as a consequence of the person's personality but may be adaptable if the individual is able to understand that this criterion is under an internal locus of control (Welch and Dawes, 2008). It is possible that tinnitus sound therapy may exert its effects not only through changing the relative distributions of tinnitus and non-tinnitus auditory activity but also through an increase in detection criterion.

\section{APPLICATION OF ALT TO TINNITUS SOUND THERAPY}

The presence of sound has been known to reduce tinnitus audibility for centuries (Stephens, 2000) with wearable tinnitus maskers being first introduced in the 1970s (Vernon and Schleuning, 1978), however, there is a great deal of debate as to the usefulness of sound as a treatment (McKenna and Irwin, 2008; Hobson et al., 2010) its modes of effect and appropriate level and type of sound (Tyler, 2006). The first sound therapy approaches attempted to relieve tinnitus by totally, or more often partially, masking the tinnitus. Acceptable tinnitus masking was believed to occur when the sound used to mask tinnitus was more tolerable than the tinnitus itself (Vernon et al., 1990; Vernon and Meikle, 2000). Recent therapies which use sound are based on habituation (Jastreboff and Hazell, 1993) or desensitization (Davis, 2006) principles and have demonstrated longer-term modifications of tinnitus reaction and perception (Henry et al., 2006; Davis et al., 2007; Bauer and Brozoski, 2011).

Alongside differing theoretical basis one of the most obvious differences in the use of sound in these therapies is the level used. Vernon et al. (1990) believed that for masking to be effective, two conditions had to be met: (1) the sound must either cover or partially cover the perception of tinnitus, (2) the masking sound must be more acceptable to the sufferer than their tinnitus sound (Vernon et al., 1990). Most clinicians believe that total masking, so that tinnitus is inaudible, is not achievable without conflicting with comfort, consequently lower levels of therapeutic sound have been advocated (Tyler, 2006). Jastreboff (1999b) proposed an effectiveness function for sound levels to be used in the habituation-based Tinnitus Retraining Therapy (TRT). The function was proposed to be governed by five factors:

(1) Stochastic resonance (enhancement of the signal by adding low-level noise); (2) dependence of the signal's strength on its contrast with the background; (3) total suppression of the signal preventing and retraining and consequently habituation; (4) partial suppression ("partial masking") which does not prevent retraining but does make it more difficult, as the training is performed on a different stimulus than the original; (5) activation of limbic and autonomic nervous systems by too loud or unpleasant sounds yielding increase of tinnitus and contracting habituation. (Jastreboff, 1999b, p. 492)

The most effective level (mixing point) governed by these five factors was proposed to occur at approximately $30 \mathrm{~dB}$ SL (Jastreboff, 1999b). Although the use of sound at the tinnitus mixing point, as described by Jastreboff (1999b), has found wide acceptance clinically, there is limited evidence at this point that it is superior (or not) to other sound levels. A greater (although not statistically significant) change in minimum masking level (MMLs) and higher percentage of patients with improvement $(40 \%$ or greater improvement in two or more questionnaires) has been shown with directive counseling combined with sound at or just below mixing level $(83.3 \%)$ compared to counseling with just audible sound (66.7\%; McKinney et al., 1999). Trials of masking versus TRT (Henry et al., 2006) and the neuromonics treatment (Tavora-Vieira et al., 2011) suggest that some people achieve greater reductions in severity with higher of levels of sound initially, with longer-term benefits with a lower level of sound. The relationship between the mixing point and MML have been examined in short-term laboratory-based evaluations (Searchfield et al., 2002; Huang et al., 2006). Huang et al. (2006) determined that the mixing point occurred at approximately $90 \%$ of MML. Searchfield et al. (2002) were able to identify a reliable mixing point in $63 \%$ (17 of 27 ) of participants at an average level of $18 \mathrm{~dB}$ SL (range 1-43.5 dB SL, $10-97.5 \%$ of the dynamic range between threshold and MML). Some of these mixing points were within the range of sounds that could theoretically result in stochastic resonance effects. The average mixing point was approximately $70 \%$ of the MML. The lowest combined tinnitus and stimulus annoyance a simple embodiment of Jastreboff (principle 5) occurred at 34\% of the range from threshold to MML. The research findings of Searchfield et al. (2002) suggest ideal therapeutic levels lower than Huang et al. (2006) and the theoretical function of Jastreboff (1999b). The experimental differences may be explained, at least in part, by the presentation methods to obtain mixing point (randomized; Searchfield et al., 2002 versus descending sequence from MML; Huang et al., 2006). The results may also not be directly comparable to Jastreboff's effectiveness function, because of the short-term nature of the experiments (Searchfield et al., 2002) versus hypothesized long-term benefit (Jastreboff, 1999b). In any case, given the limited and conflicting evidence for the benefit of one intensity of therapeutic sound over another, it would appear reasonable to suggest that further studies are required to define the optimal relationship between tinnitus and sound level (Tyler, 2006). ALT may assist in quantifying appropriate therapeutic sound levels for a given individual from a psychoacoustic point of view.

The fact that adaptation level is a weighted mean of external and internal stimuli implies that the influence of one class of stimuli may be counteracted by sufficient emphasis on other classes of stimuli. (Helson, 1964, p. 61)

The uncertainty surrounding ideal therapeutic sound parameters are not solely limited to intensity. According to ALT the level of sound is not the only consideration for tinnitus magnitude reduction; attention, arousal, personality, and other factors such as memory are critical components to the detection and audibility of tinnitus. Orientation is an important consideration in ALT (Lauterbach, 1979). The strength of orientation (extent to which change is attended to) depends on the distance between the stimulus perceived and its adaptation level. Theoretically this would imply that less orientation to tinnitus would occur when therapeutic sound is close to the adaptation level (Figure 1). This ideal level may be similar to Jastreboff's mixing point. Both neutral 
(Jastreboff, 1999b) and sound evoking emotions (such as music; Davis, 2006) have been advocated as ideal treatment sounds. A change in stimulus characteristics may result in orientation to the tinnitus signal, consequently constant signals might be advantageous, while, on the other hand, emotive (residual) factors such as stress may be reduced through pleasant music, that by its nature fluctuates. The adaptation level may also be influenced by attention to the signal (Figure 1B), hence protocols advocating simple attention training exercises to move focus away from tinnitus to other sounds or activities (Henry and Wilson, 2001; Tyler et al., 2007; Seydel et al., 2010) may result in greater adaptation, and less orientation, to the tinnitus. Tinnitus sound therapy is multifactorial and much about its optimization and modes of effect remain to be discovered. ALT offers a means to explore the contribution of emotional and attention capturing aspects of sound on tinnitus, in addition to its physical characteristics.

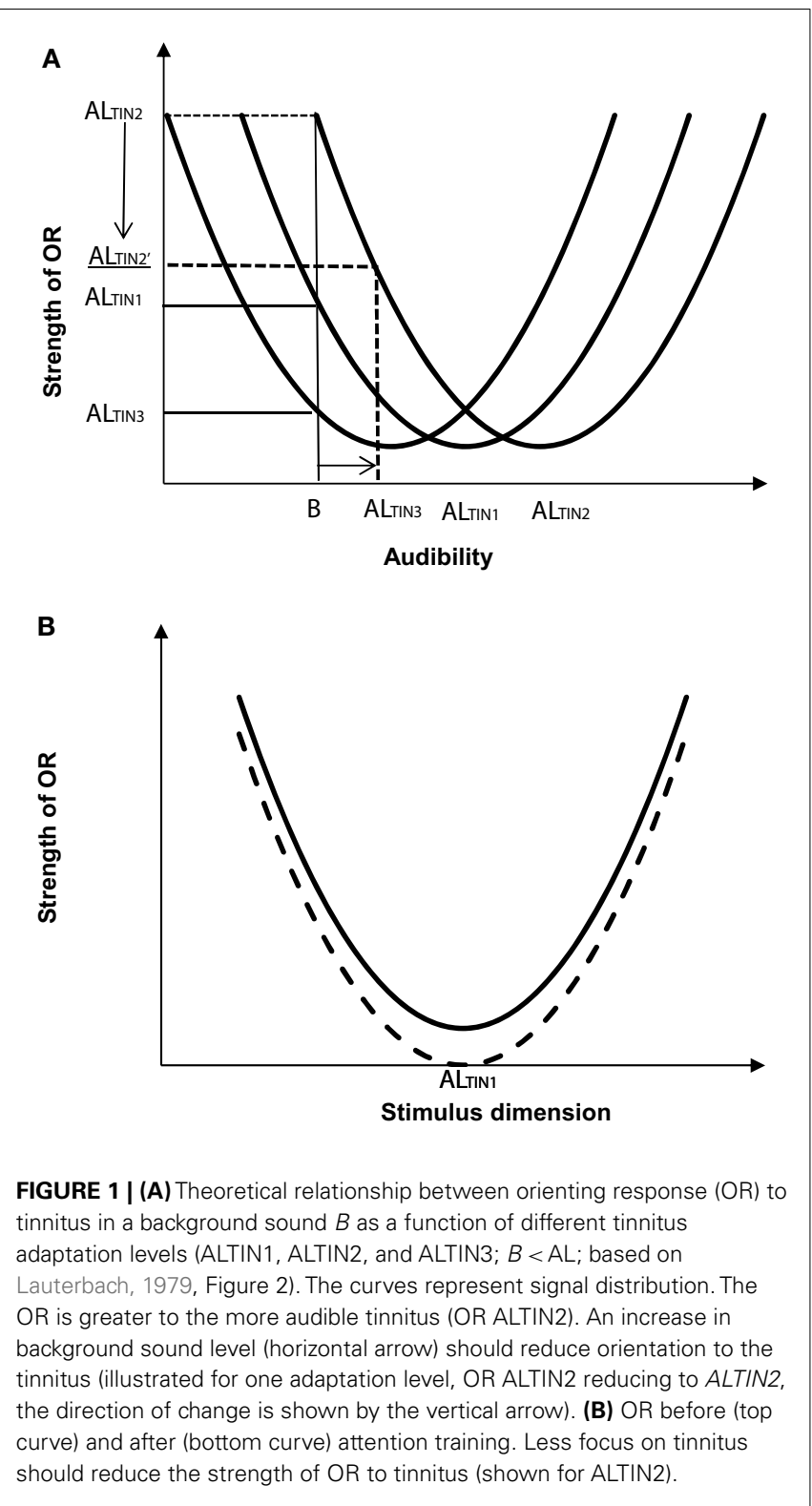

\section{SUMMARY}

In our interpretation of ALT applied to tinnitus: the intensity of the signal representing tinnitus may be constant but its contribution to tinnitus audibility and perceived magnitude will be determined by attention, background noise, and residual factors (individual's personality, depression, anxiety) all influenced by environmental factors such as arousal, adaptation level will be raised by stress, and reduced by counseling (Figure 2). Tinnitus audibility will depend both on adaptation level and contrast effects (Figure 2D). Based on our preliminary evidence and the theory of ALT we speculate that the most effective level for tinnitus sound therapy will be close to the adaptation level for tinnitus, similar to the mixing point proposed by Jastreboff (1999b); this level will vary greatly depending on individual factors and the ability (or training) of the individual to shift focus away from tinnitus to other activities, and the interaction between therapeutic sound and individual arousal.

On the one-hand ALT could be considered a simplistic interpretation of tinnitus perception, on the other hand this is its advantage, testing the theory allows the possibility of a mathematical

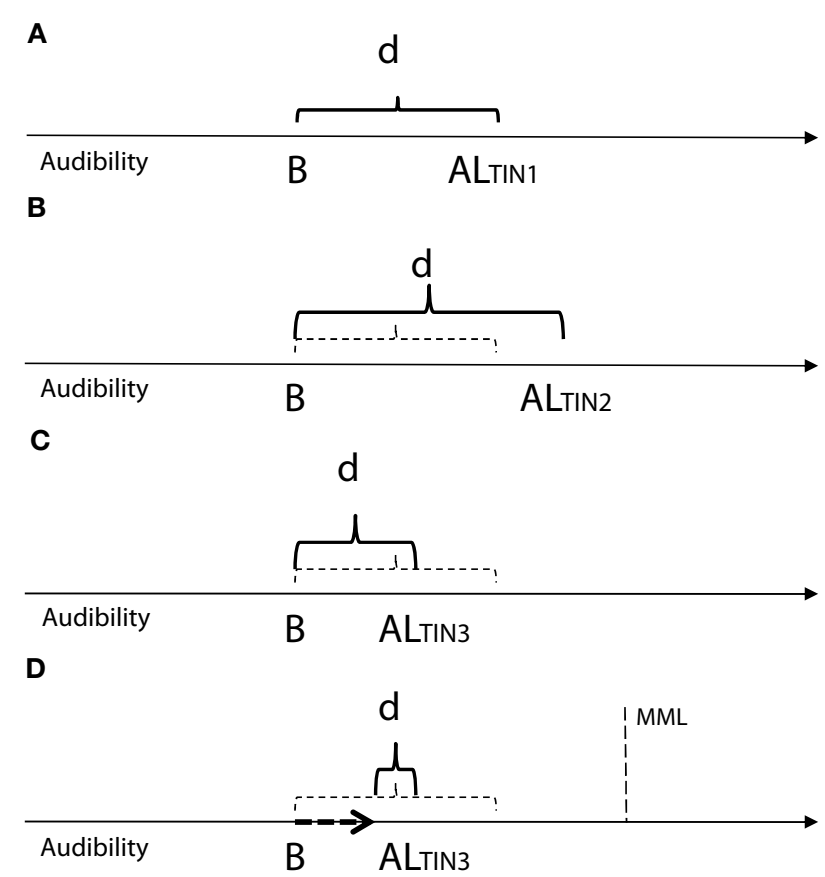

FIGURE 2 |Theoretical relationship illustrating adaptation effects on tinnitus audibility (based on Lauterbach, 1979, Figure 1). "ALTIN" is the adaptation level (center of reference) at a point in time, $B$ represents the level of background sound in the environment $(B<\mathrm{AL}), d$ is the difference between the adaptation level for tinnitus and background sound (contrast). The larger the $d$ value ( $\mathrm{AL}-B$ ) the better the perceived audibility of tinnitus. (A) Normal level of tinnitus (ALTIN1) for an individual. (B) Adaptation level (ALTIN2) raised due to emotional events, stress, anxiety (ALT's residual factor). (C) Adaptation level (ALTIN3) reduced due to management of residual factors through counseling or psychological therapy. (D) Same adaptation level (ALTIN3) with raised background sound level (representing sound therapy and counseling); tinnitus audibility $d$ is reduced due the combined effect of a lower adaptation level and reduced contrast. The minimum masking level (MML) is illustrated as an upper theoretical limit to which sound may influence the adaptation level of tinnitus. 
solution to tinnitus audibility. Whether tinnitus perception can be mathematically quantified, in the manner that weight (Helson, 1948) and temperature (Masuyama, 1994) have been, is debatable. Tinnitus is very heterogeneous and influenced by a great many factors, but that does not mean that the principles of ALT and the research methods it informs need be dismissed, merely that its implementation will require a great deal of well controlled research. ALT implies that sound therapy and counseling (or psychological management) will contribute to tinnitus audibility in the short-term, and this might be maintained by adaptive mechanisms leading to long-term reduced focus on tinnitus such as habituation. It is our intention to systematically study tinnitus perception using the ALT framework.

\section{CONCLUSION}

It has been the posit of this paper that tinnitus audibility can be explained (and mathematically expressed) as the weighted product (governed by attention and ASA) of the tinnitus signal, context (background sound), and psychological/cognitive factors (memory and personality). ALT, put simply, is a psychoacoustic, mathematical, expression of the relationship between tinnitus and the environment expressed by other authors (Jastreboff, 1999b; Tyler, 2006) but ALT enables a more analytical approach to determining the best combination of factors for a reduction in tinnitus audibility. The model has not attempted to explain the impact of

\section{REFERENCES}

Alain, C., and Arnott, S. R. (2000). Selectively attending to auditory objects. Front. Biosci. 1, D202-D212.

Andersson, G., Eriksson, J., Lundh, L. G., and Lyttkens, L. (2000). Tinnitus and cognitive interference: a Stroop paradigm study. J. Speech Lang. Hear. Res. 43, 1168-1173.

Baracca, G., Del Bo, L., and Ambrosetti, U. (2011). "Tinnitus and hearing loss," in Textbook of Tinnitus, eds A. R. Møller, B. Langguth, D. De Ridder, and T. Kleinjung (New York: Springer), 285-291.

Baskill, J. L., and Coles, R. R. A. (1999). "Relationship between tinnitus loudness and severity," in Sixth International Tinnitus Seminar, ed. J. Hazell (London: The Tinnitus and Hyperacusis Centre), 424-428.

Bauer, C. A., and Brozoski, T. J. (2011). Effect of tinnitus retraining therapy on the loudness and annoyance of tinnitus: a controlled trial. Ear Hear. 32, 145-155.

Boureau, F., Luu, M., and Doubrere, J. F. (1991). Study of experimental pain measures and nociceptive reflex in chronic pain patients and normal subjects. Pain 44, 131-138.

Bregman, A. S. (1990). Auditory Scene Analysis. The Perceptual Organization of Sound. Cambridge: MIT Press.

Broadbent, D. E., and Ladefoged, P. (1960). Vowel judgements and adaptation level. Proc. R. Soc. Lond. B Biol. Sci. 151, 384-399.

Brozoski, T. J., Wisner, K. W., Sybert, L. T., and Bauer, C. A. (2012). Bilateral dorsal cochlear nucleus lesions prevent acoustic-trauma induced tinnitus in an animal model. J. Assoc. Res. Otolaryngol. 13, 55-66.

Cherry, C. (1953). Some experiments on the recognition of speech, with one and with two ears. J. Acoust. Soc. Am. 25, 975-979.

Coren, S., and Ward, L. M. (1989). Sensation and Perception. New York: Harcourt Brace Jovanovich.

Cuny, C., Norena, A., El Massioui, F., and Chery-Croze, S. (2004). Reduced attention shift in response to auditory changes in subjects with tinnitus. Audiol. Neurootol. 9, 294-302.

Davis, P. B., Paki, B., and Hanley, P. J. (2007). Neuromonics tinnitus treatment: third clinical trial. Ear Hear. 28, 242-259.

Davis, P. B. (2006). "Music and the tinnitus," in Tinnitus Treatment, ed. R. S. Tyler (New York: Thieme), 146-160.

De Ridder, D., Elgoyhen, A. B., Romo, R., and Langguth, B. (2011). Phantom percepts: tinnitus and pain as persisting aversive memory networks. Proc. Natl. Acad. Sci. U.S.A. 108, 8075-8080.

Dean, I., Harper, N. S., and McAlpine, D. (2005). Neural population coding acoustic desensitization protocol for

tinnitus, although ALT can be applied to model affective reaction (Wilson and Gilbert, 2008). There is ample evidence for context effects in sensory perception and ALT is one example of how tinnitus perception might be studied. The theoretical relationship between tinnitus, background sound, and residual factors is consistent with evidence from population data (Welch and Dawes, 2008), observational studies (Knobel and Sanchez, 2008), and laboratory studies (Searchfield et al., 2002). ALT predicts that both sound therapy and attention training should result in a reduction in the perceived magnitude of tinnitus. However, the exact role of ALT has to be tested. Any model of tinnitus needs to be open for critique and modification as evidence becomes available. Violations from the model may be as, or more, interesting than the original theory. The neural networks involved in tinnitus, ASA, and sound therapy are complex; computation network models (Husain, 2007) are likely to make important contributions to modeling the effects of sound therapy and its further development. ALT may be a useful starting point to model tinnitus audibility networks.

\section{ACKNOWLEDGMENTS}

The authors are grateful to Manpreet Kaur, Kim Wise, and Giriraj Singh Shekhawat for their useful comments on the manuscript. The contribution of reviewers is gratefully acknowledged. The authors acknowledge the funding support of the Tinnitus Research Initiative and JM Cathie Trust.

of sound level adapts to stimulus statistics. Nat. Neurosci. 8, 1684-1689.

Dean, I., Robinson, B. L., Harper, N. S., and McAlpine, D. (2008). Rapid neural adaptation to sound level statistics. J. Neurosci. 28, 6430-6438.

Della Bitta, A. J., and Monroe, K. B. (1974). "The influence of adaptation levels on subjective price perceptions," in Advances in Consumer Research, Vol. 1, eds S. Ward and P. Wright (Urbana: Association for Consumer Research), 359-369.

Durlach, N. I., Mason, C. R., ShinnCunningham, B. G., Arbogast, T. L., Colburn, H. S., and Kidd, G. Jr. (2003). Informational masking: counteracting the effects of stimulus uncertainty by decreasing targetmasker similarity. J. Acoust. Soc. Am. $114,368-379$.

Eggermont, J. J., and Roberts, L. E. (2004). The neuroscience of tinnitus. Trends Neurosci. 27, 676-682.

Feldmann, H. (1971). Homolateral and contralateral masking of tinnitus by noise-bands and by pure tones. Audiology 10, 138-144.

Feldmann, H. (1992). “Tinnitus - reality or phantom?" in Proceedings of the Fourth International Tinnitus Seminar, ed. J. A. A. R. Dauman (Amsterdam: Kugler), 7-14.

Fishman, Y. I., and Steinschneider, M. (2010). Neural correlates of auditory scene analysis based on inharmonicity in monkey primary auditory cortex. J. Neurosci. 30, 12480-12494.

Formby, C., Sherlock, L. P., and Gold, S. L. (2003). Adaptive plasticity of loudness induced by chronic attenuation and enhancement of the acoustic background. J. Acoust. Soc. Am. 114, 55-58.

Gescheider, G. A. (1997). Psychophysics: The Fundamentals. New Jersey: Lawrence Erlbaum Associates.

Giraud, A. L., Chery-Croze, S., Fischer, G., Fischer, C., Vighetto, A., Gregoire, M. C., Lavenne, F., and Collet, L. (1999). A selective imaging of tinnitus. Neuroreport 10, 1-5.

Gopinath, B., McMahon, C. M., Rochtchina, E., Karpa, M. J., and Mitchell, P. (2010). Incidence, persistence, and progression of tinnitus symptoms in older adults: the Blue Mountains Hearing Study. Ear Hear. 31, 407-412.

Griffiths, T. D., and Warren, J. D. (2002). The planum temporale as a computational hub. Trends Neurosci. 25, 348-353.

Griffiths, T. D., and Warren, J. D. (2004). What is an auditory object? Nat. Rev. Neurosci. 5, 887-892.

Grossberg, S., Govindarajan, K. K., Wyse, L. L., and Cohen, M. A. (2004). ARTSTREAM: a neural network model of auditory scene analysis and source segregation. Neural Netw. 17, 511-536. 
Gutschalk, A., Micheyl, C., and Oxenham, A. J. (2008). Neural correlates of auditory perceptual awareness under informational masking. PLoS Biol. 6, e138. doi:10.1371/journal.pbio.0060138

Haab, L., Wallhausser-Franke, E., Trenado, C., and Strauss, D. J. (2009). Modeling limbic influences on habituation deficits in chronic tinnitus aurium. Conf. Proc. IEEE Eng. Med. Biol. Soc. 2009, 4234-4237.

Heller, M. F., and Bergman, M. (1953). Tinnitus aurium in normally hearing persons. Ann. Otol. Rhinol. Laryngol. 62, 73-83.

Helson, H. (1948). Adaptation-level as a basis for a quantitative theory of frames of reference. Psychol. Rev. 55, 297-313.

Helson, H. (1964). Adaptation-Level Theory. New York: Harper and Row.

Henry, J., and Wilson, P. H. (2001). The Psychological Management of Chronic Tinnitus: A Cognitive-Behavioural Approach. Masachusetts: Allyn \& Bacon.

Henry, J. A., and Meikle, M. A. (1996). "Loudness recruitment only partially explains the small size of tinnitus loudness-matches," in Proceedings of the Fifth International Tinnitus Seminar, eds G. E. Reich and J. A. Vernon (Portland: American Tinnitus Association), 148-157.

Henry, J. A., Schechter, M. A., Zaugg, T. L., Griest, S., Jastreboff, P. J., Vernon, J. A., Kaelin, C., Meikle, M. B., Lyons, K. S., and Stewart, B. J. (2006). Outcomes of clinical trial: tinnitus masking versus tinnitus retraining therapy. J. Am. Acad. Audiol. 17, 104-132.

Henry, J. L., and Wilson, P. H. (2002). A Self-Management Guide for the Ringing in Your Ears. Boston: Allyn \& Bacon.

Hobson, J., Chisholm, E., and El Refaie, A. (2010). Sound therapy (masking) in the management of tinnitus in adults. Cochrane Database Syst. Rev. $1-24$.

Huang, C. Y., Wu, J. L., Cheng, C. C., Sher, Y. J., and Chung, K. C. (2006). Evaluation of the mixing point in tinnitus sound therapy by a psychoacoustic matching protocol with a digital tinnitus evaluation system. ORL J. Otorhinolaryngol. Relat. Spec. $68,110-114$

Husain, F. T. (2007). Neural network models of tinnitus. Prog. Brain Res. 166, 125-140.

Jacobson, G. P., Calder, J. A., Newman, C. W., Peterson, E. L., Wharton, J. A., and Ahmad, B. K. (1996). Electrophysiological indices of selective auditory attention in subjects with and without tinnitus. Hear. Res. 97, 66-74.

Jakes, S. C., Hallam, R. S., Chambers, C. C., and Hinchcliffe, R. (1986). Matched and self-reported loudness of tinnitus: methods and sources of error. Audiology 25, 92-100.

Jastreboff, P. J. (1990). Phantom auditory perception (tinnitus): mechanisms of generation and perception. Neurosci. Res. 8, 221-254.

Jastreboff, P. J. (1999a). "The neurophysiolgical model of tinnitus and hyperacusis," in Sixth International Tinnitus Seminar, ed. J. Hazell, (London: The Tinnitus and Hyperacusis centre) 32-38.

Jastreboff, P. J. (1999b). “Optimal sound use in TRT - theory and practice," in 6th International Tinnitus Seminar, ed. J. Hazell (London: The Tinnitus and Hyperacusis Centre), 491-494.

Jastreboff, P. J., and Hazell, J. W. (1993). A neurophysiological approach to tinnitus: clinical implications. $\mathrm{Br}$. $J$. Audiol. 27, 7-17.

Jastreboff, P. J., and Hazell, J. W. P. (2004). Tinnitus Retraining Therapy: Implementing the Neurophysicological Model. Cambridge: Cambridge University Press.

Kaltenbach, J. A. (2006). The dorsal cochlear nucleus as a participant in the auditory, attentional and emotional components of tinnitus. Hear. Res. 216-217, 224-234.

Kaltenbach, J. A., Zhang, J., and Finlayson, P. (2005). Tinnitus as a plastic phenomenon and its possible neural underpinnings in the dorsal cochlear nucleus. Hear. Res. 206, 200-226.

Kidd, G. Jr., Mason, C. R., and Arbogast, T. L. (2002). Similarity, uncertainty, and masking in the identification of nonspeech auditory patterns. J. Acoust. Soc. Am. 111, 1367-1376.

Knobel, K. A., and Sanchez, T. G. (2008). Influence of silence and attention on tinnitus perception. Otolaryngol. Head Neck Surg. 138, 18-22.

Kyle, B. N., McNeil, D. W., Weinstein, B. J., and Mark, J. D. (2009). Interaction of intensity and order regarding painful events. J. Behav. Med. 32, 360-370.

Lagemann, L., Okamoto, H., Teismann, H., and Pantev, C. (2010). Bottomup driven involuntary attention modulates auditory signal in noise processing. BMC Neurosci. 11, 156. doi:10.1186/1471-2202-11-156

Lauterbach, W. (1979). Learning plus: towards a multifactorial theory of phobias - I. Adaptation level of phobic stimuli. J. Behav. Ther. Exp. Psychiatry 10, 25-28.

Lavie, N. (2005). Distracted and confused?: selective attention under load. Trends Cogn. Sci. (Regul. Ed.) 9, 75-82.

Leech, R., Gygi, B., Aydelott, J., and Dick, F. (2009). Informational factors in identifying environmental sounds in natural auditory scenes. J. Acoust. Soc. Am. 126, 3147-3155.

Marks, L. E. (1994). "Recalibrating” the auditory system: the perception of loudness. J. Exp. Psychol. Hum. Percept. Perform. 20, 382-396.

Marks, L. E., and Arieh, Y. (2006). Differential effects of stimulus context in sensory processing. Eur. Rev. Appl. Psychol. 56, 213-221.

Martin, W. H. (1995). "Spectral analysis of brain activity in the study of tinnitus," in Mechanisms of Tinnitus, eds J. A. Vernon and A. R. Møller (Boston: Allyn \& Bacon), 163-179.

Masuyama, E. (1994). Application of adaptation-level theory to human experiment on temperature sensation. Jpn. J. Erg. 30, 201-208.

Mazess, R. B. (1975). "Biological adaptation: aptitudes and acclimatization," in Biosocial Interrelations in Population Adaptation, eds E. S. Watts, F. E. Johnston, and G. W. Lasker (The Hague: Mouton Publishers), 918-928.

McBurney, D. H., and Balaban, C. D. (2009). A heuristic model of sensory adaptation. Attent. Percept. Psychophys. 71, 1941-1961.

McKenna, L., and Irwin, R. (2008). Sound therapy for tinnitus - sacred cow or idol worship?: An investigation of the evidence. Audiol. Med. 6, 16-24.

McKinney, C. J., Hazell, J. W. P., and Graham, R. (1999). "An evaluation of the TRT method," in Proceeding of the Sixth International Tinnitus Seminar, ed. J. Hazell (London: The Tinnitus and Hyperacusis centre), 99-105.

McLachlan, N., and Wilson, S. (2010). The central role of recognition in auditory perception: a neurobiological model. Psych. Rev. 117, 175-196.

Micheyl, C., Carlyon, R. P., Gutschalk, A., Melcher, J. R., Oxenham, A. J., Rauschecker, J. P., Tian, B., and Courtenay Wilson, E. (2007). The role of auditory cortex in the formation of auditory streams. Hear. Res. 229, 116-131.

Moller, A. R. (2000). Similarities between severe tinnitus and chronic pain. J. Am. Acad. Audiol. 11, 115-124.

Murch, G. M. (1973). Visual and Auditory Perception. Indianapolis: The Bobbs-Merrill Company.

Norena, A. J. (2011). An integrative model of tinnitus based on a central gain controlling neural sensitivity. Neurosci. Biobehav. Rev. 35, 1089-1109.

Oh, E. L., and Lutfi, R. A. (1999). Informational masking by everyday sounds. J. Acoust. Soc. Am. 106, 3521-3528.

Okamoto, H., Stracke, H., Stoll, W., and Pantev, C. (2010). Listening to tailormade notched music reduces tinnitus loudness and tinnitus-related auditory cortex activity. Proc. Natl. Acad. Sci. U.S.A. 19, 1207-1210.

Overmier, J. B. (2002). Sensitization, conditioning, and learning: can they help us understand somatization and disability? Scand. J. Psychol. 43, 105-112.

Penner, M. J. (1986). Magnitude estimation and the "paradoxical" loudness of tinnitus. J. Speech Hear. Res. 29, 407-412.

Penner, M. J. (1987). Masking of tinnitus and central masking. J. Speech Hear. Res. 30, 147-152.

Penner, M. J., and Bilger, R. C. (1995). "Psychoacoustical observations and the origin of tinnitus," in Mechanisms of Tinnitus, eds J. Vernon and A. R. Moller (Needham Heights: Allyn \& Bacon), 219-230.

Pressnitzer, D., Sayles, M., Micheyl, C., and Winter, I. M. (2008). Perceptual organization of sound begins in the auditory periphery. Curr. Biol. 18, 1124-1128.

Qiu, F. T., and Von Der Heydt, R. (2005). Figure and ground in the visual cortex: v2 combines stereoscopic cues with gestalt rules. Neuron 47, 155-166.

Rabinowitz, N. C., Willmore, B. D., Schnupp, J. W., and King, A. J. (2011). Contrast gain control in auditory cortex. Neuron 70, 1178-1191.

Revelle, W. (1995). "Individual differences in personality and motivation: 'non-cognitive' determinants of cognitive performance," in Attention Selection Awareness and Control. A Tribute to Donald Broadbent, eds A. Baddeley and L. Weiskrantz (Oxford: Claredon Press), 346-373.

Roberts, L. E., Eggermont, J. J., Caspary, D. M., Shore, S. E., Melcher, J. R., and Kaltenbach, J. A. (2010). Ringing ears: the neuroscience of tinnitus. $J$. Neurosci. 30, 14972-14979.

Robinson, B. L., and McAlpine, D. (2009). Gain control mechanisms in the auditory pathway. Curr. Opin. Neurobiol. 19, 402-407.

Rollman, G. B. (1979). Signal detection theory pain measures: empirical validation studies and adaptation-level effects. Pain 6, 9-21.

Scott, S. K., Rosen, S., Wickham, L. and Wise, R. J. (2004). A positron 
emission tomography study of the neural basis of informational and energetic masking effects in speech perception. J. Acoust. Soc. Am. 115, 813-821.

Searchfield, G. D., Munoz, D. J. B., and Thorne, P. R. (2004). Ensemble spontaneous activity in the guineapig cochlear nerve. Hear. Res. 192, 23-35.

Searchfield, G. D., Warr, A. A., Kuklinski, J. V., and Purdy, S. C. (2002). “Digital instruments for tinnitus: mixing point identification and thresholdadjusted noise," in Proceedings of the Seventh International Tinnitus Seminar, ed. R. Patuzzi (Perth: The University of Western Australia), 191-195.

Seydel, C., Haupt, H., Szczepek, A. J., Klapp, B. F., and Mazurek, B. (2010). Long-term improvement in tinnitus after modified tinnitus retraining therapy enhanced by a variety of psychological approaches. Audiol. Neurootol. 15, 69-80.

Shahin, A. J., Alain, C., and Picton, T. W. (2006). Scalp topography and intracerebral sources for ERPs recorded during auditory target detection. Brain Topogr. 19, 89-105.
Snyder, J. S., and Alain, C. (2007). Toward a neurophysiological theory of auditory stream segregation. Psychol. Bull. 133, 780-799.

Stallen, P. J. (2008). When exposed to sounds, would perceived loudness not be affected by social context. $J$. Acoust. Soc. Am. 123, 3690-3690.

Stephens, D. (2000). "A history of tinnitus," in Tinnitus Handbook, ed. R. Tyler (San Diego: Singular Thomson Learning), 437-449.

Tavora-Vieira, D., Eikelboom, R. H., and Miller, S. (2011). Neuromonics tinnitus treatment for patients with significant level of hearing loss: an adaptation of the protocol. Int. J. Audiol. 50, 881-886.

Tucker, D. A., Phillips, S. L., Ruth, R. A., Clayton, W. A., Royster, E., and Todd, A. D. (2005). The effect of silence on tinnitus perception. Otolaryngol. Head Neck Surg. 132, 20-24.

Tyler, R. S. (2006). "Neurophysiological models, psychological models, and treatments for tinnitus," in Tinnitus Treatment, ed. R. Tyler (New York: Thieme Medical Publishers, Inc.), $1-22$.

Tyler, R. S., Gogel, S. A., and Gehringer, A. K. (2007). Tinnitus activities treatment. Prog. Brain Res. 166, 425-434.
Vernon, J., Griest, S., and Press, L. (1990). Attributes of tinnitus and the acceptance of masking. Am. J. Otolaryngol. 11, 44-50.

Vernon, J., and Schleuning, A. (1978). Tinnitus: a new management. Laryngoscope 88, 413-419.

Vernon, J. A., and Meikle, M. B. (2000). “Tinnitus masking," in Tinnitus Handbook, ed. R. Tyler (San Diego: Singular Publishing Group), 313-357.

Watson, C. S. (2005). Some comments on informational masking. Acta Acustica United Acustica 91, 502-511.

Welch, D., and Dawes, P. J. (2008). Personality and perception of tinnitus. Ear Hear. 29, 684-692.

Wilson, T. D., and Gilbert, D. T. (2008). Explaining away. A model of affective adaptation. Pers. Psychol. Sci. 3, 370-386.

Winkler, I., Denham, S. L., and Nelken, I. (2009). Modeling the auditory scene: predictive regularity representations and perceptual objects. Trends Cogn Sci. (Regul. Ed.) 13, 532-540.

Zenner, H. P., Pfister, M., and Birbaumer, N. (2006). Tinnitus sensitization: sensory and psychophysiological aspects of a new pathway of acquired centralization of chronic tinnitus. Otol. Neurotol. 27, 1054-1063.

Zhou, X., Henin, S., Long, G. R., and Parra, L. C. (2011). Impaired cochlear function correlates with the presence of tinnitus and its estimated spectral profile. Hear. Res. 277, 107-116.

Conflict of Interest Statement: The authors declare that the research was conducted in the absence of any commercial or financial relationships that could be construed as a potential conflict of interest.

Received: 23 December 2011; accepted: 24 May 2012; published online: 13 June 2012.

Citation: Searchfield GD, Kobayashi K and Sanders $M$ (2012) An adaptation level theory of tinnitus audibility. Front. Syst. Neurosci. 6:46. doi: 10.3389/fnsys.2012.00046

Copyright (C) 2012 Searchfield, Kobayashi and Sanders. This is an open-access article distributed under the terms of the Creative Commons Attribution Non Commercial License, which permits noncommercial use, distribution, and reproduction in other forums, provided the original authors and source are credited. 